\title{
Achievable Capacity in Hybrid DS-CDMA/OFDM Spectrum-Sharing
}

\author{
Mohammad G. Khoshkholgh, Student Member, IEEE, \\ Keivan Navaie, Member, IEEE, and Halim Yanikomeroglu, Member, IEEE
}

\begin{abstract}
In this paper, we consider DS-CDMA/OFDM spectrum sharing systems and obtain the achievable capacity of the secondary service under different subchannel selection policies in the fading environment. Subchannel selection policies are divided into two categories: uniform subchannel selection, and nonuniform subchannel selection. Uniform subchannel selection is preferred for cases where a priori knowledge on subchannels state information is not available at the secondary transmitter. For cases with available a priori knowledge on subchannels state information, we study various nonuniform subchannel selection policies. In each case, we obtain the optimum secondary service power allocation and the corresponding maximum achievable capacity. Then we present results on the scaling law of the opportunistic spectrum sharing in DS-CDMA/OFDM systems with multiple users. Numerical results show that the optimal subchannel selection is based on the minimum value of the subchannel gain between the secondary transmitter and the primary receiver.
\end{abstract}

Index Terms-Dynamic spectrum access networks, DS-CDMA networks, interference threshold, OFDM, opportunistic spectrum access, spectrum sharing.

\section{INTRODUCTION}

$\mathrm{I}^{\mathrm{N}}$ spectrum sharing a Secondary Service is able to make access to a frequency band previously allocated to the Primary Service [1] and [2]. Various schemes are proposed in the literature for spectrum sharing (see, e.g., [3]). Here, our focus is on the Opportunistic Spectrum Access (OSA).

In this paper, we consider a Direct Sequence Code Division Multiple Access/Orthogonal Frequency Division Multiplexing (DS-CDMA/OFDM) spectrum sharing system in which the spectrum of a DS-CDMA-based primary service is shared with a secondary service that utilizes OFDM. DS-CDMA is the dominant air interface technique for the third generation (3G) mobile communications and some Wireless Local Area Network (WLAN) technologies. Therefore, the spectrum sharing over existing DS-CDMAbased networks is anticipated to be one of the spectrum sharing applications in the near future.

On one hand, OFDM provides the required flexibility to the secondary service to access separate underutilized portions of the spectrum band [2] and at the same time exploits the frequency selectiveness of the wireless channel. On the other hand, the spreading characteristic of DSCDMA makes it more robust to the narrow-band interference which may be imposed by spectrum sharing. Therefore, DS-CDMA/OFDM combination provides a

- M.G. Khoshkholgh and K. Navaie are with the Wireless Innovation Laboratory (WIL), Department of Electrical and Computer Engineering, Tarbiat Modares University, PO Box 14155-4843, Tehran, Iran. E-mail: \{khoshkholgh, navaie\}@modares.ac.ir.

- H. Yanikomeroglu is with the Broadband Communications and Wireless Systems (BCWS) Centre, Department of Systems and Computer Engineering, Carleton University, Ottawa, ON, Canada, K1S 5B6.

E-mail: halim@sce.carleton.ca.

Manuscript received 29 Sept. 2008; revised 2 June 2009; accepted 4 Aug. 2009; published online 5 Jan. 2010.

For information on obtaining reprints of this article, please send e-mail to: tmc@computer.org, and reference IEEECS Log Number TMC-2008-09-0384. Digital Object Identifier no. 10.1109/TMC.2010.15. new degree of freedom by enabling the secondary service to adaptively select appropriate subchannels for spectrum sharing. The benefits of this combination regarding to the maximum achievable capacity and its implementation are also studied in our previous works [4], [5], [6].

DS-CDMA systems have dynamic channel sharing and naturally are interference-limited [7]. As a metric for recognizing an underutilized portion of the primary spectrum, here, we consider a threshold on the acceptable level of the imposed interference at the primary receiver caused by the operation of the secondary users. Therefore, an under utilized portion of spectrum is defined as a frequency band in which the received interference level is below the Interference Threshold.

The subject of the present study is the maximum achievable capacity of the secondary service. The maximum achievable capacity of the secondary service for the Additive White Gaussian Channel is obtained in [8] and [9]. For flat fading environment the maximum achievable capacity of the secondary service is also obtained in [10] and [11]. In most of the related previous works in the literature, portions of the available primary spectrum are randomly selected for secondary access, see, e.g., [12] and [13].

The problem of channel assignment to multiple secondary users is also considered in [14], in which algorithms are proposed for selecting appropriate portions of the available primary spectrum based on the interference threshold constraint. Further in [15] a game theoretic approach for channel selection problem for multiple secondary users in the spectrum sharing networks is investigated.

In this paper, we propose a framework for investigating the subchannel selection policies with different objectives on the achievable capacity of the secondary service. The secondary service conducts subchannel selection based on a selection criteria. The selection criteria is a function of the corresponding subchannel gains including the channel between the secondary transmitter and the primary 
receiver, namely cross-subchannel, and the one between the secondary transmitter and receiver, which is named secondary-subchannel. We divide the subchannel selection policies into two categories: uniform subchannel selection and nonuniform subchannel selection.

In uniform subchannel selection policies, one or more subchannels are randomly selected without a priori knowledge of their corresponding secondary and crosssubchannels gains. We show that for $M$ selected subchannels, the maximum capacity is achieved by a power allocation which results in $M$ equal shares in the received interference at the primary receiver corresponding to each subchannel.

Uniform subchannel selection simply ignores the fact that some subchannels are more appropriate for OSA, i.e., a larger capacity is achieved and/or a smaller interference on the primary system is created. Therefore, we expect that taking subchannels state information into account results in a higher achieved capacity, thus a more efficient OSA. In such a scenario by considering a priori knowledge of the subchannel gains, the transmission power of the secondary service is allocated to subchannels which are more appropriate for spectrum sharing.

Intuitively, a good policy is the one that selects those subchannels which achieve the highest capacity corresponding to allocating the secondary service transmission power. Such policy selects the subchannel(s) with the highest secondary-subchannel gain for the secondary transmission. This policy may result in a higher achieved capacity for the secondary service. However, in cases where the cross-subchannel gain is also high, it creates a large interference at the primary service receiver and degrades quality-of-service (QoS) in the primary network.

Another policy may consider the level of interference that secondary service imposes at the primary receiver. Such policy may select the subchannel(s) with the lowest cross-subchannel gain for the secondary transmission. It is worth mentioning that a lower cross subchannel gain may also give the secondary service the flexibility of allocating a higher power which correspondingly results in a higher achieved capacity.

Another approach should also be envisaged in which it tries to satisfy both of the above mentioned strategies in some extent, i.e., achieving the highest possible achieved capacity and imposing the lowest possible interference at the same time.

In each case, we then formulate the OSA as an optimization problem with the objective of maximizing the achievable capacity subject to the interference threshold in the primary receiver. Finally, we obtain the optimum secondary transmit power allocation and the maximum achievable capacity through the optimization problem. Numerical results show that the optimal subchannel selection is based on the minimum value of the crosssubchannel gain.

We then investigate the spectrum sharing with multiple secondary service users and obtain the total achievable capacity of the secondary network. We first ignore the interference among the secondary service users and then obtain the achieved capacity of the secondary network with uniform and nonuniform subchannel selection scenarios and show that nonuniform subchannel selection outperforms uniform subchannel selection in multiple user case.
In the other words, nonuniform subchannel selection exploits multiuser diversity gain in the secondary network.

Taking the cross interference among the secondary users into account, we obtain the asymptote for the achieved capacity of the secondary network with uniform and nonuniform subchannel selection scenarios and show that utilizing nonuniform subchannel selection policy can result in increasing of the total achievable capacity of the secondary network by a factor of the number of subchannels.

The rest of this paper is organized as follows: In Section 2, the system model is presented; then, in Section 3, opportunistic spectrum access in DS-CDMA/OFDM is studied. In Section 4 , the achievable capacity of the secondary service with uniform subchannel selection is obtained. Then in Section 5, we analyze the achievable capacity in nonuniform subchannel selection. In Section 6, multiple secondary service users is studied. Finally, the numerical results are presented in Section 7 followed by conclusions in Section 8.

\section{System Model}

The wireless channel, we consider in this paper, is a $B \mathrm{~Hz}$ point-to-point frequency-selective Additive White Gaussian Noise (AWGN) with the power spectral density of $N_{0}$. The channel is divided into $N$ Rayleigh fading $B_{c} \mathrm{~Hz}$ subchannels where $B_{c}$ is the channel coherence bandwidth. Subchannels are indexed by $i=1,2, \ldots, N$. We assume that the subchannel gains are independent and identically distributed (i.i.d.) random processes.

We assume two services try to access the $B \mathrm{~Hz}$ spectrum band: Primary Service and Secondary Service. The frequency band has been licensed to the primary service. The secondary service does not have the spectrum license, but may acquire access to the spectrum by adopting OSA. Subscripts $s$ and $p$ are referred to the secondary service and the primary service, respectively. Hereafter, we simply refer to "primary spectrum" as "spectrum" unless otherwise stated.

The primary service utilizes DS-CDMA air interface with processing gain $G$. In this paper, our focus is the uplink. That is because most of the modern data applications are asymmetric, i.e., the amount of downlink communications is much higher than that of the uplink. Therefore, for spectrum sharing over $2 \mathrm{G}$ and/or $3 \mathrm{G}$ cellular communications the uplink spectrum is most likely underutilized, which makes it an opportunity for OSA. For a large number of users in the primary network coverage area, invoking the Central Limit Theorem justifies the Gaussian approximation for the interference process. Using second-order statistics, it is also shown that the interference process is white [16]. Therefore, the average interference in the receiver of the secondary service user in each subchannel is $(K-1) N_{0} B_{c}, K \geq 1$, where $K$ is a system parameter related to the primary network characteristics [5].

The secondary service utilizes OFDM to access the spectrum. Let $M, 0 \leq M \leq N$, be the number of accessible subchannels by the secondary service indexed by $j=$ $1, \ldots, M$. Subchannel selection is discussed in Section 3. The system we consider in this paper is time-slotted. The interference threshold, $Q$, is the maximum allowable temporal interference in the receiver of the primary service that is caused by concurrent operation of the secondary service at the same frequency band. Therefore, the secondary 


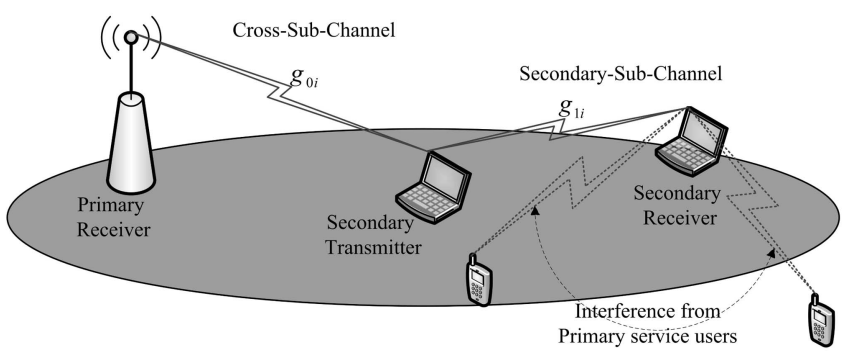

Fig. 1. The spectrum sharing structure for subchannel $i$.

service accesses to the spectrum should be managed in each time-slot so that, the interference threshold constraint is held.

The structure of the system, we consider in this paper, is depicted in Fig. 1. For subchannel $i, g_{0 i}$ and $g_{1 i}$ in Fig. 1 denote the instantaneous gains of subchannel $i$ from the secondary transmitter to the primary receiver, i.e., cross-subchannel and the secondary receiver, i.e., secondary-subchannel, respectively. Both $g_{0 i}$ and $g_{1 i}$ are assumed to be stationary and ergodic independent random variables with unit-mean and probability density functions (pdf), $f_{0 i}\left(g_{0 i}\right), f_{1 i}\left(g_{1 i}\right)$, respectively. Channel gains $g_{0 i}$ and $g_{1 i}$ are i.i.d. for $i=1,2, \ldots, N$. In our analysis, we assume that the perfect Channel Side Information (CSI) pair $\left(g_{0 i}, g_{1 i}\right), i=1, \ldots, N$ is available at the transmitters. ${ }^{1}$ In practise, the CSI pair may be made available through a spectrum coordinator or by proper signalling. Note that the results derived based on this assumption act as an upper-bound for the cases without perfect CSI pair.

Since our focus is on the secondary service maximum achievable capacity, similar to the spectrum sharing literature (see, e.g., [10], [9]), we start our analysis considering one secondary user. In cases where more than one secondary users are competing to access to the underutilized frequency bands, the total secondary service achievable capacity is upper-bounded by the case with only one secondary user. This is due to the fact that, secondary users also impose interference on each other. Interference is imposed because of imperfectness in actual multiple access techniques utilized in the secondary network. We later extend our analysis into the multiple secondary service users in Section 6.

\section{Opportunistic Spectrum Sharing in DS-CDMA Primary NetWorks}

At a given time instant, we define a policy $\mathcal{P}_{\Psi}$ based on a deterministic selection criteria $\Psi(.,$.$) and set$

$$
\mu_{i} \triangleq \Psi\left(g_{0 i}, g_{1 i}\right)
$$

Corresponding to observed random variables $\mu_{i}, i=1, \ldots, N$, we define the selection sequence

$$
\Upsilon_{N}=\left(\mu_{r_{1}}, \mu_{r_{2}}, \ldots, \mu_{r_{N}}\right) \triangleq \mathcal{P}_{\Psi}\left(\mu_{1}, \mu_{2}, \ldots, \mu_{N}\right) .
$$

The $N$-tuple selection sequence is arranged so that its first element, indexed by $r_{1}$, is the most suitable subchannel for spectrum sharing based on the selection criteria in (1). Adopting a new indexing for brevity, we also define the M-tuple selected sequence

1. Channel side information (CSI) contains the probability distribution of the channel gain, as well as its actual value at a certain time instant.

$$
\Theta_{M}=\left(\theta_{1}, \theta_{2}, \ldots, \theta_{M}\right) \triangleq\left(\mu_{r_{1}}, \mu_{r_{2}}, \ldots, \mu_{r_{M}}\right) .
$$

We assume that the pdf of random variable $\theta_{j}$ is $k_{j}(\theta)$, $j=1, \ldots, M$. Note that, based on such policy if $\theta_{j_{1}}$ and $\theta_{j_{2}}$ are entities in the selected sequence and $j_{1}<j_{2}$, then the corresponding subchannel with index $j_{1}$ is considered more suitable for spectrum sharing comparing to that of $j_{2}$.

If $\Psi\left(g_{0 i}, g_{1 i}\right)$ is constant, noting that $g_{0 i}$ and $g_{1 i} \forall i$ are i.i.d. random variables, thus, $\mu_{i}$ and consequently $\theta_{j}, j=1, \ldots, M$ are i.i.d.. In other words, subchannels are considered uniform and $M$ out of $N$ subchannels are selected randomly without any a priori knowledge on their status. We call this selection strategy uniform subchannel selection. For cases with variable $\Psi\left(g_{0 i}, g_{1 i}\right)$, different subchannels based on the corresponding values of $\Psi\left(g_{0 i}, g_{1 i}\right)$ are treated nonuniformly. We call this selection strategy as nonuniform subchannel selection.

The instantaneous transmission power of the secondary service over the $j$ th subchannel is $P_{s j}\left(g_{0 j}\right)$ which we refer to as $P_{s j}$. We set $\mathbf{P}_{s}=\left(P_{s 1}, \ldots, P_{s M}\right)$ as the secondary service transmission power vector over $M$ subchannels.

Assume that the secondary service communicates over the selected subchannel $j$ with transmission power of $P_{s j}$. Narrow-band interference denoted by $Q_{j}$ is correspondingly imposed at the front-end of the primary service receiver, where

$$
Q_{j}=g_{0 r_{j}} P_{s j}
$$

Since the air interface in the primary network is DS-CDMA, the narrow-band interference $Q_{j}$ is then spread out over the whole $B \mathrm{~Hz}$ bandwidth and manifests itself as an equivalent wide-band interference equal to $G^{-1} Q_{j}$ at the primary receiver.

For $M$ accessible subchannels, the secondary service transmits with the transmission power vector $\mathbf{P}_{s}=\left(P_{s 1}\right.$, $\left.P_{s 2}, \ldots, P_{s M}\right)$. Therefore, the equivalent narrow-band interference $\mathbf{Q}=\left(Q_{1}, Q_{2}, \ldots, Q_{M}\right)$ is implied at the front-end of the primary receiver. Consequently, to comply with the interference threshold, $Q$, we should have

$$
\frac{1}{G} \sum_{j=1}^{M} g_{0 r_{j}} P_{s j} \leq Q .
$$

For a given $Q$, the maximum achievable capacity of the secondary service, for $M$ selected subchannels based on policy $\mathcal{P}_{\Psi}, C_{s \mid M}^{\Psi}$, is the solution of the following optimization problem:

Problem $\mathcal{O} 1$.

$$
\begin{gathered}
C_{s \mid M}^{\Psi}=\max _{\mathbf{P}_{s}} \sum_{j=1}^{M} B_{c} \int_{g_{1 r_{j}}, g_{0 r_{j}}} \log \left(1+\frac{g_{1 r_{j}} P_{s j}}{K N_{0} B_{c}}\right) \\
\times f_{1 j}\left(g_{1 r_{j}}\right) f_{0 j}\left(g_{0 r_{j}}\right) d g_{0 r_{j}} d g_{1 r_{j}}, \\
\text { s.t. } \quad \frac{1}{G} \sum_{j=1}^{M} g_{0 r_{j}} P_{s j} \leq Q, \\
\sum_{j=1}^{M} P_{s j} \leq P_{s},
\end{gathered}
$$


where (6) is Shannon's Capacity formula corresponding to power vector $\mathbf{P}_{s},(7)$ is the interference threshold, (8) is the secondary service maximum transmit power constraint, and $P_{s}$ is the secondary service maximum transmit power. ${ }^{2}$

In practice, the interference threshold constraint is usually tight enough so that the transmit power constraint for the secondary service does not meet; therefore, for clarity of expositions, similar to the related literature (see, e.g., [10]), we do not consider the transmit power constraint for the secondary service in (8). In cases where the transmit power constraint is the dominant constraint comparing to the interference threshold, it is shown in [4] that the achieved capacity without considering transmit power constraint serves as an upper-bound. The optimization problem in $\mathcal{O} 1$ is an instant of water-filling problem (for water-filling problem see, e.g., [16]).

\section{Uniform Subchannel Selection}

Let $\Psi\left(g_{0 i}, g_{1 i}\right)=1$, thus $\mathcal{P}_{1}$ return $\theta_{j}, j=1, \ldots M$ which are i.i.d.. As it was mentioned, in uniform subchannel selection, subchannels are considered uniformly and $M$ out of $N$ subchannels are selected randomly by $\mathcal{P}_{1}$ without any a priori knowledge on their status.

The probability of selecting a subchannel in uniform subchannel selection scenario is thus equal to $1 / N$. Substituting $P_{s j}=\frac{Q_{j}}{g_{0 r_{j}}}, j=1,2, \ldots, M$ and defining

$$
\gamma_{Q_{j}} \triangleq \frac{Q_{j}}{K N_{0} B_{c}}
$$

$\mathcal{O} 1$ is converted into the following:

Problem $\mathcal{O} 2$.

$$
\begin{aligned}
& C_{s \mid M}^{1}= \max _{\mathbf{Q}} \sum_{j=1}^{M} B_{c} \int_{\nu_{r_{j}}} \log \left(1+\nu_{r_{j}} \gamma_{Q_{j}}\right) h_{j}\left(\nu_{r_{j}}\right) d \nu_{r_{j}}, \\
& \text { s.t. } \quad \sum_{j=1}^{M} Q_{j}=G Q, \quad 0 \leq Q_{j} \leq G Q,
\end{aligned}
$$

where we define reward factor, $\nu_{r_{j}}$, as

$$
\nu_{r_{j}} \triangleq \frac{g_{1 r_{j}}}{g_{0 r_{j}}}, \quad 0<\nu_{r_{j}}<\infty
$$

In cases where subchannel gains $\sqrt{g_{0 i}}$ and $\sqrt{g_{1 i}}$ are i.i.d. Rayleigh random variables, $g_{0 i}$ and $g_{1 i}$ are exponentially distributed random variables; therefore, the pdf of $\nu_{r_{j}}$ is

$$
\begin{aligned}
h_{j}\left(\nu_{r_{j}}\right) & =\frac{d}{d \nu_{r_{j}}} \int_{0}^{\infty} \int_{0}^{g_{0 r_{j}} \nu_{r_{j}}} e^{-g_{0 r_{j}}} e^{-g_{1 r_{j}}} d g_{0 r_{j}} d g_{1 r_{j}} \\
& =\int_{0}^{\infty} g_{0 r_{j}} e^{-g_{0 r_{j}}\left(1+\nu_{r_{j}}\right)} d g_{0 r_{j}},
\end{aligned}
$$

or, equivalently,

$$
h_{j}\left(\nu_{r_{j}}\right)=\frac{1}{\left(1+\nu_{r_{j}}\right)^{2}}, \quad 0<\nu_{r_{j}}<\infty .
$$

2. In practice, CDMA cellular systems are single frequency; therefore, the operation of the secondary service in the primary band may impose unexpected interference on the base-stations of the adjacent cells. To deal with this issue, one may add new constraints to the optimization Problem $\mathcal{O} 1$ or consider a conservative value for $Q$. Hereafter, for brevity we consider the latter case.

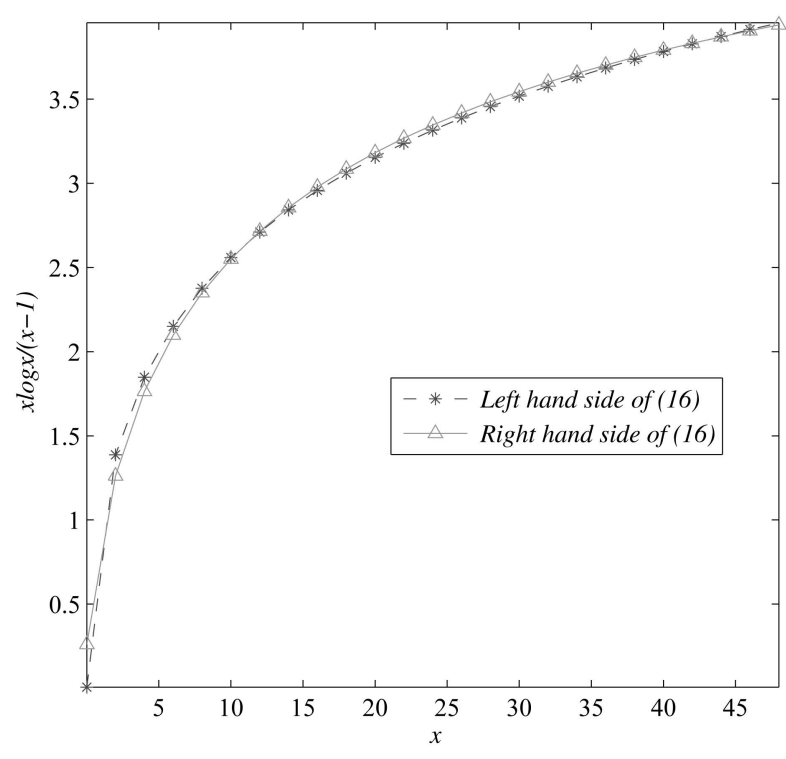

Fig. 2. Pseudolinear approximation for $A_{1}=-1.2015, A_{2}=-0.0052$, $A_{3}=1.0772, A_{4}=3.0262$, and $A_{5}=3.8829$.

By substituting (12) into (10), and integrating by part, $\mathcal{O} 2$ is simplified as follows:

Problem $\mathcal{O} 3$.

$$
\begin{aligned}
C_{s \mid M}^{1}= & \max _{\underline{\gamma_{Q}}} \sum_{j=1}^{M} B_{c} \frac{\gamma_{Q_{j}}}{\gamma_{Q_{j}}-1} \log \left(\gamma_{Q_{j}}\right), \\
& \text { s.t. } \quad \sum_{j=1}^{M} \gamma_{Q_{j}}=G N \gamma_{Q}, \quad 0 \leq \gamma_{Q_{j}} \leq G N \gamma_{Q},
\end{aligned}
$$

where we define $\gamma_{Q}$ as the spectrum sharing load factor:

$$
\gamma_{Q} \triangleq \frac{Q}{K N_{0} B}
$$

and, correspondingly, $\underline{\gamma_{Q}}=\left(\gamma_{Q_{1}}, \gamma_{Q_{2}}, \ldots, \gamma_{Q_{M}}\right)$ as the spectrum sharing load vector.

\subsection{Pseudolinear Approximation}

To find an approximate solution for $\mathcal{O} 3$ we replace (13) by the following pseudolinear approximation:

$$
\frac{x}{x-1} \log (x) \approx A_{1}+A_{2} x+A_{3} \log \left(A_{4} x+A_{5}\right) .
$$

Fig. 2 shows the left and the right hand sides of (16) for $A_{1}=-1.2015, A_{2}=-0.0052, A_{3}=1.0772, A_{4}=3.0262$, and $A_{5}=3.8829$. As it is seen in this figure, the approximation in (16) follows the approximated function very closely.

Using Lagrange's multipliers approach together with the pseudolinear approximation in (16), the Lagrangian Function corresponding to $\mathcal{O} 3$ is

$$
\begin{aligned}
\left.L \underline{\left(\gamma_{Q}\right.}, \lambda\right)= & \sum_{j=1}^{M} A_{1}+A_{2} \gamma_{Q_{j}}+A_{3} \log \left(A_{4} \gamma_{Q_{j}}+A_{5}\right) \\
& -\lambda\left(\sum_{j=1}^{M} \gamma_{Q_{j}}-G N \gamma_{Q}\right)
\end{aligned}
$$


where $\lambda$ is the Lagrangian coefficient. By differentiating $L\left(\gamma_{Q}, \lambda\right)$ with respect to $\gamma_{Q_{j}}$ and setting this derivative equal to zero, we obtain

$$
\gamma_{Q_{j}}^{*}=\frac{A_{3}}{\lambda^{*}-A_{2}}-\frac{A_{5}}{A_{4}} .
$$

Substituting (17) into (14), we have

$$
\sum_{j=1}^{M}\left[\frac{A_{3}}{\lambda^{*}-A_{2}}-\frac{A_{5}}{A_{4}}\right]=G N \gamma_{Q}
$$

hence,

$$
\lambda^{*}=A_{2}+\frac{A_{3}}{\frac{G N \gamma_{Q}}{M}+\frac{A_{5}}{A_{4}}} .
$$

Consequently, substituting (19) into (17) yields

$$
\gamma_{Q_{j}}^{*}=\frac{G N \gamma_{Q}}{M}, \quad j=1,2, \ldots, M .
$$

Note that (20) suggests that for given $M$, and $Q$, the maximum achievable capacity is obtained by dividing the total acceptable interference, $G N \gamma_{Q}$, into $M$ equal portions for each subchannel, which in fact is a direct consequence of selecting $M$ out of $N$ subchannel without any a priori knowledge.

Correspondingly, using (9) and (4) the optimal transmission power vector, $\mathbf{P}_{s}^{*}$, is obtained as follows:

$$
\mathbf{P}_{s}^{*}=\left(\frac{1}{g_{0 r_{1}}} \frac{G Q}{M}, \frac{1}{g_{0 r_{2}}} \frac{G Q}{M}, \ldots, \frac{1}{g_{0 r_{M}}} \frac{G Q}{M}\right) .
$$

Equation (21) shows that the interference share for each selected subchannel $j, \gamma_{Q_{j}}^{*}$, is mapped into the corresponding transmission power, $P_{s j}^{*}$, proportional to $1 / g_{0 r_{j}}$. Therefore, if $g_{0 r_{j}}$ is large, then the secondary user's transmission creates a large interference at the primary service receiver. In this case, (21) suggests a lower secondary transmission power in selected subchannel $j$.

The maximum achievable capacity of the secondary service is approximated by substituting (20) into (13):

$$
C_{s \mid M}^{1} \approx M B_{c} \frac{G N \gamma_{Q}}{G N \gamma_{Q}-M} \log \left(\frac{G N \gamma_{Q}}{M}\right) .
$$

For a practical case in which $Q=G^{-1} N_{0} B$ and $M \ll N$, the entries of the spectrum sharing load vector can be obtained from (20) as $\gamma_{Q_{j}}=\frac{N}{K M}$ which are much higher than unity. ${ }^{3}$

\section{Nonuniform Subchannel Selection}

Uniform subchannel selection simply ignores the fact that some subchannels are more appropriate for OSA because of their corresponding CSIs, i.e., a larger capacity is achieved and/or a smaller interference on the primary users is created. Therefore, we expect that nonuniform subchannel selection based on a priori knowledge of the secondarysubchannel and/or cross-subchannel CSIs, results in a higher secondary service achieved capacity.

3. In practice, $K$ adopts a moderate value within the range of 2-8 [5]; obviously, systems with a lower $K$ are more suitable for spectrum sharing.
Intuitively, an appropriate policy could consider the interference that the secondary service activity creates at the primary receiver. Such policy may select the subchannel(s) with the lowest cross-subchannel gain(s), $g_{0 i}$, for the secondary transmission which creates a lower interference on the primary receiver. Potentially, a lower $g_{0 i}$ may also give the secondary service the flexibility of allocating a higher power which correspondingly results in a higher achieved capacity. This policy is called cross-subchannelbased selection policy. To implement cross-subchannel-based selection policy, the secondary service requires $g_{0 i}$ during each time-slot. To obtain $g_{0 i}$, a direct or indirect (i.e., through a third party such as spectrum manager) signaling channel between the primary service receiver (i.e., basestation) and the secondary service transmitter is required.

Another option is the one that selects those subchannels which achieve the highest capacity corresponding to allocating the secondary service transmit power. Such policy selects the subchannel(s) with the highest secondarysubchannel gain, $g_{1 i}$, for the secondary transmission. This policy is called secondary-subchannel-based selection policy.

Another approach could also be envisaged in which subchannel selection policy tries to employ a combination of the aforementioned two strategies in some sense, i.e., achieving the highest possible achieved capacity while imposing the lowest possible interference at the same time. As an instance of such combination, we define $g_{1 i} / g_{0 i}$ as the reward factor of subchannel $i$ and call the corresponding subchannel selection policy as the reward factor-based subchannel selection policy. In this section, we obtain the maximum achievable capacity for three aforementioned subchannel selection policies.

\subsection{Cross-Subchannel-Based Subchannel Selection}

Assume that the selection criteria is

$$
\Psi\left(g_{0 i}, g_{1 i}\right)=g_{0 i},
$$

and correspondingly, $\mu_{i}=g_{0 i}$. Policy $\mathcal{P}_{g_{0}}$ in then defined so that, in the $N$-tuple selection sequence, $\Upsilon_{N}$,

$$
\mu_{r_{1}} \leq \mu_{r_{2}} \leq \cdots \leq \mu_{r_{N}}
$$

and

$$
\mu_{r_{1}} \triangleq \min _{i}\left\{\mu_{i}\right\}
$$

The $M$-tuple selected sequence, $\Theta_{M}$, is then defined as

$$
\theta_{1}=\mu_{r_{1}} \leq \theta_{2}=\mu_{r_{2}} \leq \cdots \leq \theta_{M}=\mu_{r_{M}} .
$$

The main objective of $\mathcal{P}_{g_{0}}$ is to select the subchannel(s) which cause(s) the lowest imposed interference at the primary receiver. The pdf of $\theta_{j}, \forall j$, is obtained using order statistics (see, e.g., [17]):

$$
k_{j}(\theta)=N_{j} F_{\mu}^{j-1}(\theta)\left[1-F_{\mu}(\theta)\right]^{N-j} f_{\mu}(\theta),
$$

where

$$
N_{j} \triangleq \frac{N !}{(j-1) !(N-j) !},
$$

and $f_{\mu}(\theta)$ and $F_{\mu}(\theta)$ are pdf and probability distribution function (PDF) of the random variable $\mu$, respectively. By following the same argument as in Section 4 for Rayleigh 
fading, $f_{\mu}(\theta)=e^{-\theta}$, and $F_{\mu}(\theta)=1-e^{-\theta}$. Thus, $k_{j}(\theta)$ is obtained as following:

$$
k_{j}(\theta)=N_{j}\left(1-e^{-\theta}\right)^{j-1} e^{-\theta(N-j+1)} .
$$

Replacing the binomial expansion of $\left(1-e^{-\theta}\right)^{j-1}$ in (25),

$$
k_{j}(\theta)=N_{j} \sum_{l=0}^{j-1} F_{l}^{j-1} e^{-\theta(N-l)},
$$

where $F_{l}^{j-1}$ is defined as

$$
F_{l}^{j-1} \triangleq\left(\begin{array}{c}
j-1 \\
l
\end{array}\right)(-1)^{j-1-l}
$$

Proposition 1. The maximum achievable capacity of the secondary service based on policy $\mathcal{P}_{g_{0}}, C_{s \mid M}^{g_{0}}$, is obtained from the following optimization problem:

Problem $\mathcal{O} 4$.

$$
\begin{aligned}
C_{s \mid M}^{g_{0}}= & \max _{\underline{\underline{\gamma_{Q}}}} \sum_{j=1}^{M} \sum_{l=0}^{j-1} B_{c} N_{j} F_{l}^{j-1} \frac{\gamma_{Q_{j}} \log \left((N-l) \gamma_{Q_{j}}\right)}{(N-l) \gamma_{Q_{j}}-1} \\
& \text { s.t. } \quad \sum_{j=1}^{M} \gamma_{Q_{j}}=G N \gamma_{Q}, \quad 0 \leq \gamma_{Q_{j}} \leq G N \gamma_{Q} .
\end{aligned}
$$

\section{Proof. See Appendix A.}

Note that in practice $M \ll N$, thus, $N \gamma_{Q_{j}} \gg 1$. Consequently $\mathcal{O} 4$ is approximated by the following optimization problem:

\section{Problem $\mathcal{O} 5$.}

$$
\begin{aligned}
& C_{s \mid M}^{g_{0}} \approx \max _{\underline{\gamma_{Q}}} \sum_{j=1}^{M} \sum_{l=0}^{j-1} B_{c} \frac{N_{j} F_{l}^{j-1}}{N-l} \log \left((N-l) \gamma_{Q_{j}}\right), \\
& \text { s.t. } \quad \sum_{j=1}^{M} \gamma_{Q_{j}}=G N \gamma_{Q}, \quad 0 \leq \gamma_{Q_{j}} \leq G N \gamma_{Q} .
\end{aligned}
$$

The Lagrangian function corresponding to $\mathcal{O} 5$ is

$$
\begin{aligned}
\left.L \underline{\gamma_{Q}}, \lambda\right)= & \sum_{j=1}^{M} \sum_{l=0}^{j-1} \frac{N_{j} F_{l}^{j-1}}{N-l} \log \left((N-l) \gamma_{Q_{j}}\right) \\
& -\lambda\left(\sum_{j=1}^{M} \gamma_{Q_{j}}-G N \gamma_{Q}\right),
\end{aligned}
$$

where $\lambda$ is the Lagrangian coefficient. By differentiating $L\left(\gamma_{Q}, \lambda\right)$ with respect to $\gamma_{Q_{j}}$ and setting this derivative equal to zero, we obtain:

$$
\gamma_{Q_{j}}^{*}=\frac{1}{\lambda^{*}} \vartheta_{j},
$$

where we define

$$
\vartheta_{j} \triangleq \sum_{l=0}^{j-1} N_{j} \frac{F_{l}^{j-1}}{N-l}
$$

Substituting (32) into (31),

$$
\lambda^{*}=\frac{1}{G N \gamma_{Q}} \sum_{j=1}^{M} \vartheta_{j}
$$

The optimal spectrum sharing load factor, $\gamma_{Q_{j}}^{*}$, is then obtained by substituting (33) into (32) as follows:

$$
\gamma_{Q_{j}}^{*}=G N \gamma_{Q} \frac{\vartheta_{j}}{\sum_{j=1}^{M} \vartheta_{j}} .
$$

Correspondingly, using (9) and (4), the optimal transmission power vector, $\mathbf{P}_{s}^{*}$, is

$$
\mathbf{P}_{s}^{*}=\frac{G Q}{\sum_{j=1}^{M} \vartheta_{j}}\left(\frac{\vartheta_{1}}{g_{0 r_{1}}}, \frac{\vartheta_{2}}{g_{0 r_{2}}}, \ldots, \frac{\vartheta_{M}}{g_{0 r_{M}}}\right) .
$$

The maximum achievable capacity of the secondary service is approximated by substituting (34) into (30) as

$$
C_{s \mid M}^{g_{0}} \approx \sum_{j=1}^{M} \sum_{l=0}^{j-1} \frac{B_{c} N_{j} F_{l}^{j-1}}{N-l} \log \left((N-l) G N \gamma_{Q} \frac{\vartheta_{j}}{\sum_{j=1}^{M} \vartheta_{j}}\right)
$$

For $M=1$, the approximated achievable capacity in (36) reduces to

$$
C_{s \mid 1}^{g_{0}} \approx B_{c} \log \left(G N^{2} \gamma_{Q}\right)
$$

which is very close to the exact solution of $\mathcal{O} 5$ for $M=1$ that is

$$
C_{s \mid 1}^{g_{0}}=B_{c} \frac{G N^{2} \gamma_{Q}}{G N^{2} \gamma_{Q}-1} \log \left(G N^{2} \gamma_{Q}\right)
$$

\subsection{Secondary-Subchannel-Based Subchannel Selection}

Assume that the selection criteria is

$$
\Psi\left(g_{0 i}, g_{1 i}\right)=g_{1 i},
$$

and correspondingly, $\mu_{i}=g_{1 i}$. Policy $\mathcal{P}_{g_{1}}$ in then defined so that, in the $N$-tuple selection sequence, $\Upsilon_{N}$,

$$
\mu_{r_{N}} \leq \mu_{r_{N-1}} \leq \cdots \leq \mu_{r_{1}}
$$

and

$$
\mu_{r_{1}} \triangleq \max _{i}\left\{\mu_{i}\right\}
$$

The $M$-tuple selected sequence, $\Theta_{M}$, is then defined as

$$
\theta_{M}=\mu_{r_{M}} \leq \theta_{M-1}=\mu_{r_{M-1}} \leq \cdots \leq \theta_{1}=\mu_{r_{1}} .
$$

Here, $\mathcal{P}_{g_{1}}$ selects those subchannels which result in the highest achieved capacity for the secondary service. Again, using order statistics, pdf of $\theta_{j}$ is obtained as

$$
k_{j}(\theta)=N_{j} F_{\mu}^{N-j}(\theta)\left[1-F_{\mu}(\theta)\right]^{j-1} f_{\mu}(\theta),
$$

where $f_{\mu}(\theta)$ and $F_{\mu}(\theta)$ are pdf and probability distribution function (PDF) of the random variable $\mu$, respectively. Following the same line of argument as in Section 4, for Rayleigh fading, $k_{j}(\theta)$ is obtained as the following:

$$
k_{j}(\theta)=N_{j}\left(1-e^{-\theta}\right)^{N-j} e^{-\theta j} .
$$

Replacing the binomial expansion of $\left(1-e^{-\theta}\right)^{N-j}$ in (39),

$$
k_{j}(\theta)=N_{j} \sum_{l=0}^{N-j} F_{l}^{N-j} e^{-\theta(l+j)},
$$




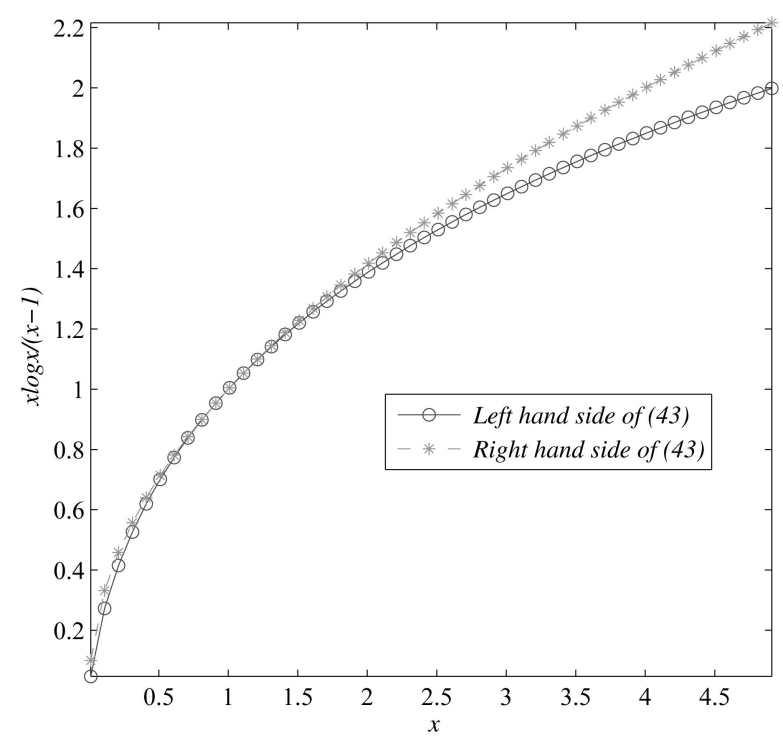

Fig. 3. Approximation in (43).

where $N_{j}$ is obtained from (24) and

$$
F_{l}^{N-j} \triangleq\left(\begin{array}{c}
N-j \\
l
\end{array}\right)(-1)^{l}
$$

Proposition 2. The maximum achievable capacity of the secondary service based on policy $\mathcal{P}_{g_{1}}, C_{s \mid M^{\prime}}^{g_{1}}$ is obtained from following optimization problem:

\section{Problem $\mathcal{O} 6$.}

$$
\begin{array}{r}
C_{s \mid M}^{g_{1}}=\max _{\underline{\gamma_{\mathbf{Q}}}} \sum_{j=1}^{M} \sum_{l=0}^{N-j} B_{c} \frac{N_{j} F_{l}^{N-j}}{l+j} \frac{\frac{\gamma_{Q_{j}}}{l+j}}{\frac{\gamma_{Q_{j}}}{l+j}-1} \log \left(\frac{\gamma_{Q_{j}}}{l+j}\right), \\
\text { s.t. } \quad \sum_{j=1}^{M} \gamma_{Q_{j}}=G N \gamma_{Q}, \quad 0 \leq \gamma_{Q_{j}} \leq G N \gamma_{Q} .
\end{array}
$$

Proof. See Appendix B.

For small values of $\frac{\gamma_{Q_{j}}}{l+j}, l=0,1, \ldots, N-j$, we utilize the following approximation (see Fig. 3):

$$
\frac{x}{x-1} \log x \approx \sqrt{x}
$$

which reduces Problem $\mathcal{O} 6$ into the following:

\section{Problem $\mathcal{O} 7$.}

$$
\begin{aligned}
& C_{s \mid M}^{g_{1}} \approx \max _{\underline{\underline{Q}}} \sum_{j=1}^{M} \sum_{l=0}^{N-j} B_{c} \frac{N_{j} F_{l}^{N-j}}{l+j}\left(\frac{\gamma_{Q_{j}}}{l+j}\right)^{\frac{1}{2}}, \\
& \text { s.t. } \quad \sum_{j=1}^{M} \gamma_{Q_{j}}=G N \gamma_{Q}, \quad 0 \leq \gamma_{Q_{j}} \leq G N \gamma_{Q} .
\end{aligned}
$$

Similar to $\mathcal{O} 5$, utilizing Lagrange's multipliers for solving $\mathcal{O} 7$, the optimal spectrum sharing load factor $\gamma_{Q_{j}}^{*}$ is obtained as follows:

$$
\gamma_{Q_{j}}^{*}=G N \gamma_{Q} \frac{\chi_{j}^{2}}{\sum_{j=1}^{M} \chi_{j}^{2}}
$$

where

$$
\chi_{j} \triangleq \sum_{l=0}^{N-j} N_{j} \frac{F_{l}^{N-j}}{2(l+j)^{1.5}} .
$$

Correspondingly, using (9) and (4), the optimal transmission power vector, $\mathbf{P}_{s}^{*}$, is

$$
\mathbf{P}_{s}^{*}=\frac{G Q}{\sum_{j=1}^{M} \chi_{j}^{2}}\left(\frac{\chi_{1}^{2}}{g_{0 r_{1}}}, \frac{\chi_{2}^{2}}{g_{0 r_{2}}}, \ldots, \frac{\chi_{M}^{2}}{g_{0 r_{M}}}\right) .
$$

The maximum achievable capacity of the secondary service is then approximated by substituting (46) into (44),

$$
C_{s \mid M}^{g_{1}} \approx \sum_{j=1}^{M} \sum_{l=0}^{N-j} B_{c} \frac{N_{j} F_{l}^{N-j}}{(l+j)^{1.5}}\left(G N \gamma_{Q} \frac{\chi_{j}^{2}}{\sum_{j=1}^{M} \chi_{j}^{2}}\right)^{\frac{1}{2}} .
$$

For $M=1$, the approximated achievable capacity in (48) is reduced to

$$
C_{s \mid 1}^{g_{1}} \approx B_{c} \sum_{l=0}^{N-1} N \frac{F_{l}^{N-1}}{l+1}\left(\frac{G N \gamma_{Q}}{l+1}\right)^{\frac{1}{2}},
$$

which, noting (43), it is close to the exact solution of Problem $\mathcal{O} 8$, that is

$$
C_{s \mid 1}^{g_{1}}=B_{c} \sum_{l=0}^{N-1} N \frac{F_{l}^{N-1}}{l+1} \frac{\frac{G N \gamma_{Q}}{l+1}}{\frac{G N \gamma_{Q}}{l+1}-1} \log \left(\frac{G N \gamma_{Q}}{l+1}\right) .
$$

\subsection{Reward Factor-Based Subchannel Selection}

Assume that the selection criteria is

$$
\Psi\left(g_{0 i}, g_{1 i}\right) \triangleq \nu_{i}=\frac{g_{1 i}}{g_{0 i}},
$$

and correspondingly, $\mu_{i}=\nu_{i}$. Policy $\mathcal{P}_{\nu}$ in then defined so that, in the $N$-tuple selection sequence, $\Upsilon_{N}$,

$$
\mu_{r_{N}} \leq \mu_{r_{N-1}} \leq \cdots \leq \mu_{r_{1}}
$$

and

$$
\mu_{r_{1}} \triangleq \max _{i}\left\{\mu_{i}\right\}
$$

The $M$-tuple selected sequence, $\Theta_{M}$, is then defined as following:

$$
\theta_{M}=\mu_{r_{M}} \leq \theta_{M-1}=\mu_{r_{M-1}} \leq \cdots \leq \theta_{1}=\mu_{r_{1}} .
$$

Using order statistics, the pdf $k_{j}(\theta)$ is obtained as follows:

$$
k_{j}(\theta)=N_{j} H_{\mu}^{N-j}(\theta)\left[1-H_{\mu}(\theta)\right]^{j-1} h_{\mu}(\theta),
$$

where $H_{\mu}(\theta), h_{\mu}(\theta)$ are the pdf the PDF of random variable $\mu$, respectively.

For cases in which $\sqrt{g_{0 j}}$ and $\sqrt{g_{1 j}}$ are i.i.d., and have Rayleigh distribution, $\forall j$, it was already shown that $h_{\mu}(\theta)$ is (see Section 4)

$$
h_{\mu}(\theta)=\frac{1}{(1+\theta)^{2}}, \quad 0<\theta<\infty
$$

and $H_{\mu}(\theta)$ is 


$$
H_{\mu}(\theta)=\frac{\theta}{(1+\theta)}, \quad 0<\theta<\infty
$$

Substituting (53) and (52) into (51),

$$
k_{j}(\theta)=N_{j} \frac{\theta^{N-j}}{(1+\theta)^{N+1}}, \quad 0<\theta<\infty,
$$

where $N_{j}$ is obtained from (24).

Substituting $P_{s j}=\frac{Q_{j}}{g_{0 r_{j}}}$ into (6), and setting $\theta_{j}=\frac{g_{1 r_{j}}}{g_{0 r_{j}}}$, the maximum achievable capacity $C_{s \mid M}^{\nu}$ based on policy $\mathcal{P}_{\nu}$ is obtained from $\mathcal{O} 1$ through the following optimization problem:

Problem $\mathcal{O} 8$.

$$
\begin{aligned}
C_{s \mid M}^{\nu}= & \max _{\underline{\underline{Q}}} \sum_{j=1}^{M} B_{c} \int_{0}^{\infty} \log \left(1+\theta_{j} \gamma_{Q_{j}}\right) k_{j}\left(\theta_{j}\right) d \theta_{j}, \\
& \text { s.t. } \quad \sum_{j=1}^{M} \gamma_{Q_{j}}=G N \gamma_{Q}, \quad 0 \leq \gamma_{Q_{j}} \leq G N \gamma_{Q} .
\end{aligned}
$$

Since obtaining a closed form solution for $C_{s \mid M}^{\nu}$ in $\mathcal{O} 8$ is complicated mainly, due to the form of pdf, $k_{j}(\theta)$, in (55), in this paper, we obtain $C_{s \mid M}^{\nu}$ utilizing numerical results. Note that, for $M=1$, (55) is reduced to

$$
C_{s \mid 1}^{\nu}=B_{c} \int_{0}^{\infty} \log \left(1+\theta G N \gamma_{Q}\right) k_{1}(\theta) d \theta .
$$

\section{Multiple Secondary Service Users}

Here, we consider the case where more than one secondary service transmitter-receiver pairs are communicating using OSA. The secondary network consists of a number of secondary service users which employ OSA to access the spectrum. The access technology of the secondary network is OFDM. Let $N_{s}$ be the number of secondary service active transmitter-receiver pairs, indexed by $s$ each with the corresponding spectrum sharing load factor $\gamma_{s}, s=1, \ldots, N_{s}$. In the following, we first simply assume that each secondary service transmitter-receiver pair selects only one subchannel utilizing a subchannel selection scenario. We then extend our analysis to the case where each secondary service transmitter-receiver pair selects multiple subchannels.

The equivalent created wide-band interference corresponding to each secondary service transmitter-receiver pair is equal to $Q_{s}=K N_{0} B \gamma_{s}$. Since our main objective is to obtain the maximum achievable capacity, for brevity of discussions we ignore the interfering effect of different secondary service transmitter-receiver pairs on each other. The interfering effect, if any, reduces the achieved capacity of the secondary network, thus our obtained results act as an upper-bound.

\subsection{Uniform Subchannel Selection}

Consider the case where only one subchannel is selected for each secondary service transmitter-receiver pair utilizing uniform subchannel selection. Therefore, from (22) the maximum achievable capacity for the secondary service $s$ is

$$
C_{s}^{1}=B_{c} \frac{G N \gamma_{s}}{G N \gamma_{s}-1} \log \left(G N \gamma_{s}\right)
$$

Total achievable capacity of the secondary network $C^{1}$, is then obtained from the following optimization problem:

Problem $\mathcal{O} 9$.

$$
\begin{array}{r}
C^{1}=\max _{\underline{\gamma_{S}}} \sum_{s=1}^{N_{s}} B_{c} \frac{G N \gamma_{s}}{G N \gamma_{s}-1} \log \left(G N \gamma_{s}\right), \\
\text { s.t. } \quad \sum_{s=1}^{N_{s}} \gamma_{s}=\gamma_{Q}, \quad 0 \leq \gamma_{s} \leq \gamma_{Q},
\end{array}
$$

where $\gamma_{S} \triangleq\left(\gamma_{1}, \ldots, \gamma_{N_{s}}\right)$. Following the same line of arguments $\overline{\overline{a s}}$ in Section 4.1, the optimal spectrum sharing load factor $\gamma_{s}^{*}$ is

$$
\gamma_{s}^{*}=\frac{\gamma_{Q}}{N_{s}}
$$

The total achievable capacity of the secondary network is then obtained by substituting (61) into (59) as

$$
C^{1}=B_{c} N_{s} \frac{G N \gamma_{Q}}{G N \gamma_{Q}-N_{s}} \log \left(\frac{G N \gamma_{Q}}{N_{s}}\right) .
$$

\subsection{Nonuniform Subchannel Selection}

We also consider the case that nonuniform subchannel selection based on policy $\mathcal{P}_{g_{0}}$ is employed for the secondary service. In this case, the maximum achievable capacity of the secondary service transmitter-receiver pair $s$ with one subchannel selection is obtained from (37) as

$$
C_{s \mid 1}^{g_{0}}=B_{c} \frac{G N^{2} \gamma_{s}}{G N^{2} \gamma_{s}-1} \log \left(G N^{2} \gamma_{s}\right)
$$

The total achievable capacity of the secondary network, $C^{g_{0}}$, is obtained from the following optimization problem:

Problem $\mathcal{O} 10$.

$$
\begin{aligned}
& C^{g_{0}}=\max _{\underline{\gamma_{S}}} \sum_{s=1}^{N_{s}} B_{c} \frac{G N^{2} \gamma_{s}}{G N^{2} \gamma_{s}-1} \log \left(G N^{2} \gamma_{s}\right), \\
& \text { s.t. } \quad \sum_{s=1}^{N_{s}} \gamma_{s}=\gamma_{Q}, \quad 0 \leq \gamma_{s} \leq \gamma_{Q} .
\end{aligned}
$$

In this case, similar to Section 6.1 the optimal spectrum sharing load factor, $\gamma_{s}^{*}$, is also obtained from (61). Intuitively, from the secondary network point of view each user shares an equal spectrum sharing load factor because each secondary service transmitter-receiver pair selects one subcannel based on policy $\mathcal{P}_{g_{0}}$.

Substituting (61) into (64), the total achievable capacity of the secondary network is obtained as

$$
C^{g_{0}}=B_{c} N_{s} \frac{G N^{2} \gamma_{Q}}{G N^{2} \gamma_{Q}-N_{s}} \log \left(\frac{G N^{2} \gamma_{Q}}{N_{s}}\right) .
$$

\subsection{Impact of Inter Secondary Service Interference}

In practice, in the secondary network, the transmission made by a secondary service transmitter-receiver pair, also interferes the other active secondary service transmitterreceiver pairs. To understand the scaling effect of the 
achievable capacity of the secondary network we assume that $N_{s} \gg N$.

Suppose that the secondary service transmitter-receiver pairs are fixed communications entities. In such case adopting the result from [18] the total achievable capacity of the secondary network tends to zero with increasing $N_{s}$ by rate $1 / \sqrt{N_{s}}$. Therefore, for uniform subchannel selection

$$
\lim _{N_{s} \rightarrow \infty} C^{1} \propto B_{c} \sqrt{N_{s}} \frac{G N \gamma_{Q}}{G N \gamma_{Q}-N_{s}} \log \left(\frac{G N \gamma_{Q}}{N_{s}}\right),
$$

and for nonuniform subchannel selection,

$$
\lim _{N_{s} \rightarrow \infty} C^{g_{0}} \propto B_{c} \sqrt{N_{s}} \frac{G N^{2} \gamma_{Q}}{G N^{2} \gamma_{Q}-N_{s}} \log \left(\frac{G N^{2} \gamma_{Q}}{N_{s}}\right) .
$$

Therefore,

$$
\lim _{N_{s} \rightarrow \infty} \frac{C^{g_{0}}}{C^{1}} \propto N
$$

Equation (69) shows that increasing the number of secondary service users, the total achievable capacity of the secondary network by policy $\mathcal{P}_{g_{0}}$ is $N$ time higher than that of $\mathcal{P}_{1}$.

For the case where the secondary service users are mobile and delay tolerant, adopting the results in [19] the decreasing rate of the total achievable capacity of the secondary network can be kept constant. Under the assumption of the mobility along with the infinite delay tolerant both uniform and nonuniform subchannel selections are able to achieve the corresponding achievable capacity in (62), and (66), respectively.

\subsection{Multiple Subchannel Selection}

We extend our previous analysis to the case where each secondary service transmitter-receiver pair selects multiple subchannels. We consider the case where the inter secondary service interference is ignorable. Taking the inter secondary service interference into account is straightforward using the similar approach adopted in Section 6.3.

Let each secondary service transmitter-receiver pair select $M$ subchannels. In the uniform subchannel selection policy, using (22) the optimization problem, $\mathcal{O} 9$, is converted to the following:

Problem $\hat{\mathcal{O}} 9$.

$$
\begin{gathered}
C_{M}^{1}=\max _{\underline{\underline{\gamma_{S}}}} \sum_{s=1}^{N_{s}} M B_{c} \frac{G N \gamma_{s}}{G N \gamma_{s}-M} \log \left(\frac{G N \gamma_{s}}{M}\right), \\
\text { s.t. } \quad \sum_{s=1}^{N_{s}} \gamma_{s}=\gamma_{Q}, \quad 0 \leq \gamma_{s} \leq \gamma_{Q} .
\end{gathered}
$$

Let us define $\hat{\gamma}_{s} \triangleq \gamma_{s} / M$; therefore, the above optimization problem can be easily rewritten as follows:

Problem $\hat{\hat{\mathcal{O}}} 9$.

$$
\begin{gathered}
C_{M}^{1}=\max _{\hat{\gamma}_{S}} M \sum_{s=1}^{N_{s}} B_{c} \frac{G N \hat{\gamma}_{s}}{G N \hat{\gamma}_{s}-1} \log \left(G N \hat{\gamma}_{s}\right), \\
\text { s.t. } \quad \sum_{s=1}^{N_{s}} \hat{\gamma}_{s}=\frac{\gamma_{Q}}{M}, \quad 0 \leq \hat{\gamma}_{s} \leq \gamma_{Q} .
\end{gathered}
$$

Consequently, similar to $\mathcal{O} 9$ the optimal value of $\hat{\gamma}_{s}^{*}$ is

$$
\hat{\gamma}_{s}^{*}=\frac{\gamma_{Q}}{M N_{s}}
$$

which is similar to the optimal spectrum sharing load factor $\gamma_{s}^{*}=\gamma_{Q} N_{s}^{-1}$ in (61). The total achievable capacity of the secondary network is then obtained by substituting $\gamma_{s}^{*}$ into (70) as

$$
C_{M}^{1}=B_{c} M N_{s} \frac{G N \gamma_{Q}}{G N \gamma_{Q}-M N_{s}} \log \left(\frac{G N \gamma_{Q}}{M N_{s}}\right) .
$$

For the nonuniform multiple subchannels selection based on policy $\mathcal{P}_{g_{0}}$, using (36), the optimization problem $\mathcal{O} 10$ is converted to the following:

Problem $\hat{\mathcal{O}} 10$.

$$
\begin{aligned}
C_{M}^{g_{0}}= & \max _{\underline{\underline{\gamma_{S}}}} \sum_{s=1}^{N_{s}} \sum_{j=1}^{M} \sum_{l=0}^{j-1} \frac{B_{c} N_{j} F_{l}^{j-1}}{N-l} \\
& \times \log \left((N-l) G N \gamma_{s} \frac{\vartheta_{j}}{\sum_{j=1}^{M} \vartheta_{j}}\right), \\
\text { s.t. } & \sum_{s=1}^{N_{s}} \gamma_{s}=\gamma_{Q}, \quad 0 \leq \gamma_{s} \leq \gamma_{Q},
\end{aligned}
$$

where $N_{j}$ and $F_{l}^{j-1}$ are obtained from (24) and (27), respectively, and $\vartheta_{j}$ is defined as follows:

$$
\vartheta_{j} \triangleq \sum_{l=0}^{j-1} N_{j} \frac{F_{l}^{j-1}}{N-l}
$$

Utilizing Lagrange's multipliers approach for solving $\hat{\mathcal{O}} 10$, the optimal spectrum sharing load factor, $\gamma_{s}^{*}$, is also obtained from (61). Consequently, the total achievable capacity of the secondary network is then obtained by substituting $\gamma_{s}^{*}$ into (71) as

$$
C_{M}^{g_{0}}=\sum_{j=1}^{M} \sum_{l=0}^{j-1} \frac{N_{s} B_{c} N_{j} F_{l}^{j-1}}{N-l} \log \left((N-l) G N \frac{\gamma_{Q}}{N_{s}} \frac{\vartheta_{j}}{\sum_{j=1}^{M} \vartheta_{j}}\right) .
$$

\section{Numerical Results}

We consider $\gamma_{Q}=-30 \mathrm{~dB}$, and $N=40$. In this section, $\lambda_{0}$ and $\lambda_{1}$ denote the mean values of channel gains $g_{0 r_{j}}$ and $g_{1 r_{j}}$, respectively. Here, we compare the achievable capacity of different subchannel selection policies under different scenarios.

\subsection{Comparing Subchannel Selection Policies}

First, we compare the achieved spectral efficiency of the subchannel selection policies versus $M$ for $\lambda_{1}=\lambda_{0}$. We consider two cases. In the first case, for a fixed value of interference threshold, the number of accessible subchannels, $M$, is increased. In the second case, corresponding to increasing $M$, we also increase the interference threshold with the same rate, thus, the spectrum sharing load factor, $\gamma_{Q}$, is also increased by scaling factor $M$. For easy reference, here we again define four subchannel selection policies; $\mathcal{P}_{1}$ : uniform subchannel selection policy, $\mathcal{P}_{g_{0}}$ : subchannel selec- 


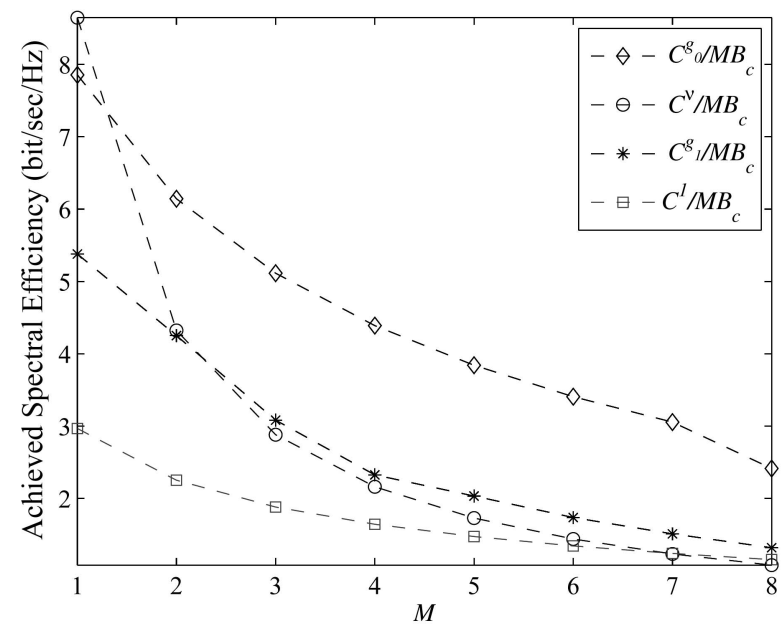

Fig. 4. Achieved spectral efficiency of the secondary service for various subchannel selection policies versus $M$, for $\gamma_{Q}=-30 \mathrm{~dB}$, and $N=40$, and $\lambda_{1}=\lambda_{0}$.

tion policy based on the cross-subchannel, $\mathcal{P}_{g_{1}}$ : subchannel selection policy based on the secondary-subchannel, and $\mathcal{P}_{\nu}$ : reward factor-based subchannel selection policy.

For the case of constant interference threshold, as it is seen in Fig. 4, the achieved spectral efficiency of uniform subchannel selection, $C_{s \mid M}^{1} / M B_{c}$, is lower than that of nonuniform case in most cases. For $M=1$, the gap in the achieved spectral efficiency between $C_{s \mid M}^{1} / M B_{c}$ and $C_{s \mid M}^{\nu} / M B_{c}$ is very large. However, by increasing $M$, this gap is significantly reduced. This gap is related to the ratio $M / N$, for larger values of this ratio this gap is lower. This is mainly due to the fact that for larger $M / N$, the set of $M$ selected subchannels by $\mathcal{P}_{\nu}$ and $\mathcal{P}_{1}$ have probably large overlap.

It is also seen that $\mathcal{P}_{g_{1}}$ performs very similar to $\mathcal{P}_{\nu}$ for larger values of $M$. Comparing the rate of decreasing the achieved spectral efficiency by increasing $M$ indicates that $C_{s \mid M}^{g_{0}} / M B_{c}$ is decreased with a slower rate than that of the others. The slower decay rate is mainly due to the fact that considering the cross-subchannel gain, $g_{0 i}$, lets the secondary transmitter send the maximum transmit power which is also generates less interference on the receiver of the primary service, and holds the interference threshold constraint. In most cases, the maximum transmit power by $\mathcal{P}_{g_{0}}$ can be larger than that of the others, thus, the corresponding achievable capacity is higher.

Fig. 4 indicates that when comparing the achieved spectral efficiency for a given number of accessible subchannels to the secondary service, $\mathcal{P}_{g_{0}}$ outperforms the other subchannel selection policies.

For the case that the interference threshold is also increased with the same ratio as $M$, Fig. 5 indicates that in uniform subchannel selection, the achieved spectral efficiency remains constant when the number of accessible subchannels, $M$, is increased. Indeed, in uniform subchannel selection, $\gamma_{Q}$ is equally divided into $M$ subchannels, (see, (20)); therefore, the increase in $\gamma_{Q}$ neutralizes the impact of increasing $M$. For nonuniform subchannel selection policies, increasing $M$ results in decreasing the achieved spectral efficiency; however, the observed decrease is smaller than that of seen in Fig. 4.

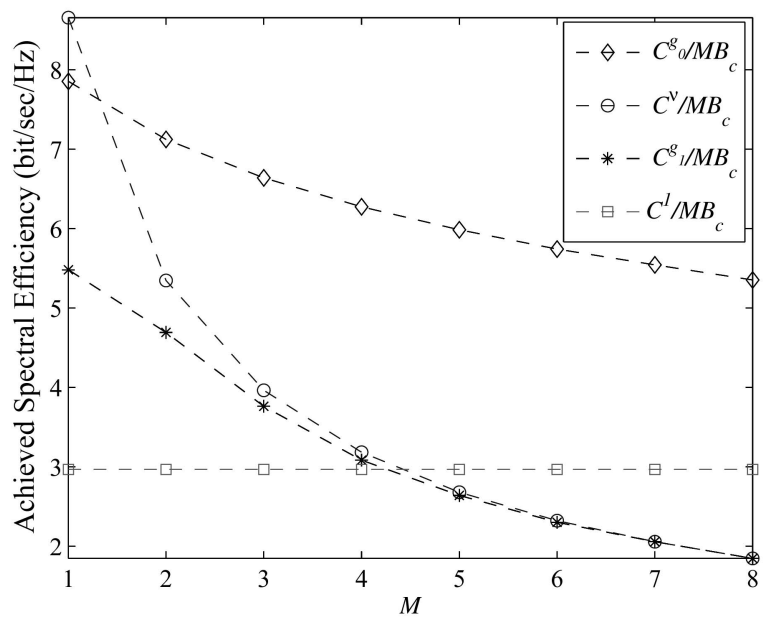

Fig. 5. Achieved spectral efficiency of the secondary service for various subchannel selection policies versus $M$, for $N=40$, and $\lambda_{1}=\lambda_{0}$.

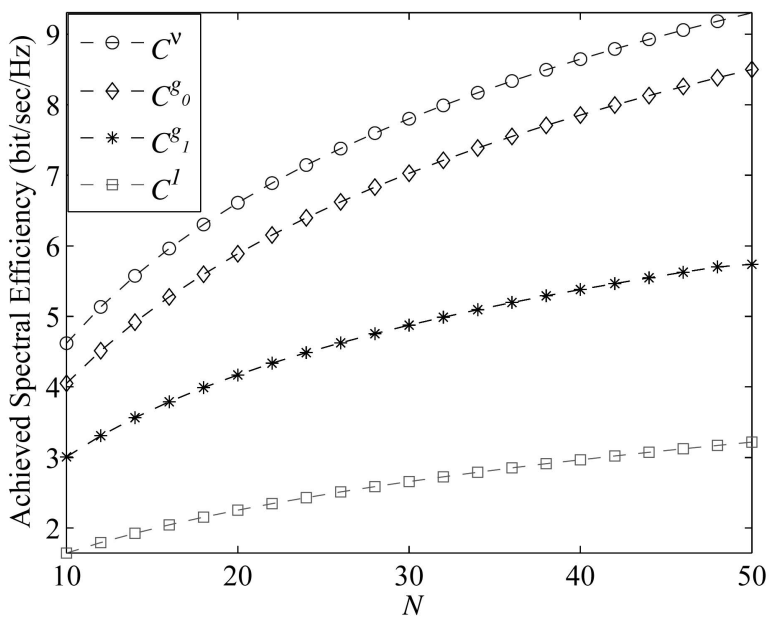

Fig. 6. Achieved spectral efficiency of the secondary service for various subchannel selection policies versus $N$, for $\gamma_{Q}=-30 \mathrm{~dB}$, and $M=1$, and $\lambda_{1}=\lambda_{0}$.

An interesting point in Fig. 5 is that the rate of decaying of the achieved spectral efficiency for $\mathcal{P}_{g_{0}}$ by increasing $M$, is much lower than that of the others and even lower than that of the case with constant interference threshold in Fig. 4. It is also seen that for some values of $M, \mathcal{P}_{1}$ outperforms $\mathcal{P}_{g_{1}}$, and $\mathcal{P}_{\nu}$. This is due to the fact that with increasing $M / N$, the probability that $\mathcal{P}_{1}$ selects some subchannels with small $g_{0 r_{j}}$ or large $g_{1 r_{j}}$ is increased. Note that finding a subchannel $\mathcal{P}_{1}$ can allocates a larger transmit power than that of $\mathcal{P}_{g_{1}}$ and $\mathcal{P}_{\nu}$. Therefore, the achieved spectral efficiency is increased.

To study the impact of $N$, in Fig. 6, we compare the achieved spectral efficiency of the secondary service for $M=1$ versus $N$ for different subchannel selection policies when $\lambda_{1}=\lambda_{0}$. As it is expected, for both uniform and nonuniform subchannel selection policies, the achieved spectral efficiency is increased by increasing $N$. This is due to the fact that the probability of selecting proper subchannel for OSA is increased by increasing $N$. The rate of increment in the achieved spectral efficiency for $\mathcal{P}_{\nu}$ and $\mathcal{P}_{g_{0}}$ follows the same pattern and have a larger increasing rate when compared to $\mathcal{P}_{1}, \mathcal{P}_{g_{1}}$. It is also interesting to note that, by increasing $N$ the gap between the achieved spectral efficiency of $\mathcal{P}_{g_{0}}$ and $\mathcal{P}_{1}$ is also increased. 


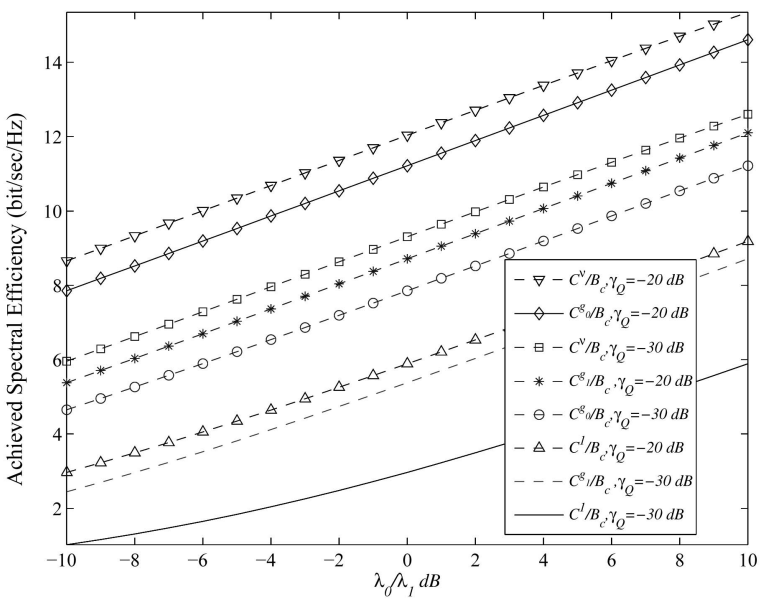

Fig. 7. Achieved spectral efficiency of the secondary service for various subchannel selection policies versus $\frac{\lambda_{0}}{\lambda_{1}}$, for $M=1, N=40$.

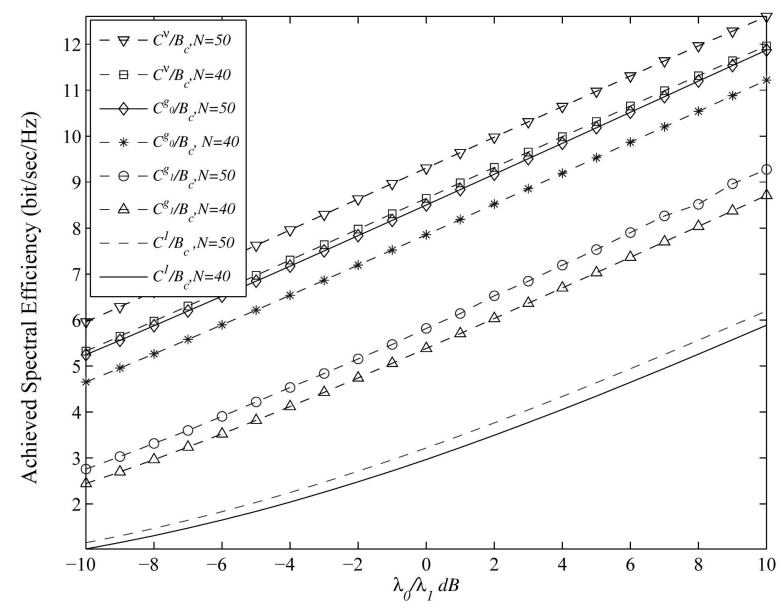

Fig. 8. Achieved Spectral efficiency of the secondary service for various subchannel selection methods versus $\frac{\lambda_{0}}{\lambda_{1}}$ for $M=1$.

Here, we also study the impact of the cross-subchannel, $g_{0 j}$, and the secondary subchannel, $g_{1 j}$, on the achieved spectral efficiency of different subchannel selection policies. In Fig. 7 , the achieved spectral efficiency is given versus $\lambda_{0} / \lambda_{1}$, for different values of spectrum sharing load factor, $\gamma_{Q}$ and $N=40$. By increasing $\lambda_{0} / \lambda_{1}$, the attenuation of $g_{0 r j}$ is increased and $g_{1 r_{j}}$ is decreased, i.e., the impact of fading in cross-subchannel is increased comparing to the secondary subchannel. Therefore, by increasing $\lambda_{0} / \lambda_{1}$, the achieved spectral efficiency is also increased. By increasing $\gamma_{Q}$, the achieved spectral efficiency for all subchannel selection policies is also increased.

Fig. 8 shows the impact of $N$ for various subchannel selection with different fading situations, and $\gamma_{Q}=-30 \mathrm{~dB}$. As it is seen, by increasing $N$, the achieved spectral efficiency is increased.

\subsection{Impact of Multiple Secondary Users}

First, we simply ignore the interference between the secondary service transmitter-receiver pairs. In Fig. 9, the total achievable capacity of the secondary network versus the number of the secondary service users, $N_{s}$, is given. As it is seen, for uniform subchannel selection for $N_{s}<\left\lfloor G N \gamma_{Q}\right\rfloor$, $C^{1}$ is increased versus $N_{s}$ in an approximately linear

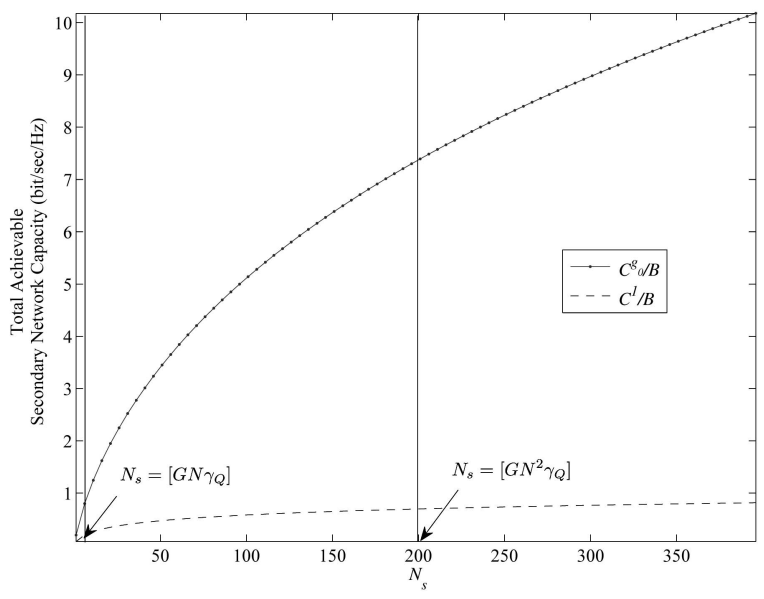

Fig. 9. Total achievable capacity of the secondary network versus $N_{s}$, for uniform and nonuniform subchannel selection without considering cross interference among the secondary service users $\left(\gamma_{Q}=-30 \mathrm{~dB}\right.$, and $N=40)$.

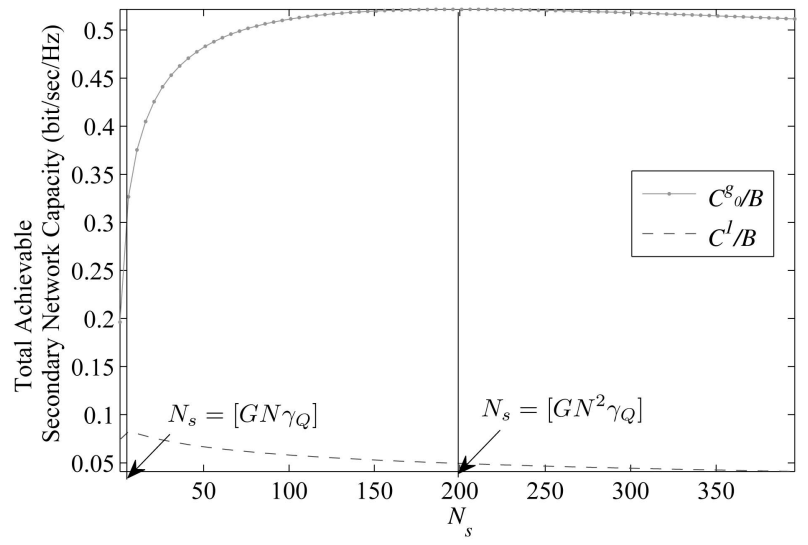

Fig. 10. Total achievable capacity of the secondary network versus $N_{s}$, for uniform and nonuniform subchannel selection with considering cross interference among the secondary service users $\left(\gamma_{Q}=-30 \mathrm{~dB}\right.$, and $N=40)$.

fashion, where $\lfloor x\rfloor$ is the largest integer smaller than $x$. The total achievable capacity of the secondary network, $C^{1}$, remains constant for $N_{s} \gg\left\lfloor G N \gamma_{Q}\right\rfloor$. For subchannel selection policy $\mathcal{P}_{g_{0}}$, however, by increasing $N_{s}$ the total achievable capacity of the secondary network, $C^{g_{0}}$, is significantly increased comparing to $C^{1}$. The observed behavior has the same root as multiuser diversity gain [20].

In Fig. 10, we plot the asymptote of the achieved capacity as we obtained in Section 6.3. As it is seen, for large values of $N_{s}, C^{1}$ is very close to zero. In nonuniform subchannel selection for $0 \leq N_{s} \leq\left\lfloor G N^{2} \gamma_{Q}\right\rfloor, C^{g_{0}}$ is increased versus $N_{s}$. For $N_{s}=\left\lfloor G N^{2} \gamma_{Q}\right\rfloor$ the maximum secondary service achievable capacity in the secondary network is $C^{g_{0}}=\sqrt{G N^{2} \gamma_{Q}}$, which can also be obtained by taking derivation of (68) and setting the results equal to zero. Therefore, although the number of the secondary service users in the network is increased, the total achievable capacity of the secondary network is constant in nonuniform subchannel selection for $N_{s}>\left\lfloor G N^{2} \gamma_{Q}\right\rfloor$. 


\section{Conclusions}

In this paper, the achievable capacity of the secondary service in DS-CDMA/OFDM spectrum sharing was studied where the access of the secondary service is OFDM and the primary service air interface is DS-CDMA. The achievable capacity of the secondary service based on different subchannel selection policies are obtained. We considered two main category of subchannel selection policies including uniform subchannel selection and nonuniform subchannel selection. It is seen that in the uniform subchannel selection the achieved capacity is maximized when the power is allocated to each subchannel so that the corresponding received interference at the primary receiver is equal for all subchannels. In nonuniform subchannel selection based on a priori knowledge of subchannel gains, a proper set of subchannels for OSA is selected. As it was shown, the achievable capacity of the secondary service based on nonuniform subchannel selection is increased versus uniform subchannel selection.

For the case that the number of accessible subchannel was only one, the numerical results confirmed that the best policy for subchannel selection was $\mathcal{P}_{\nu}$. However, by increasing the accessible subchannels, the subchannel selection policy based on $\mathcal{P}_{g_{0}}$ was dominant. Since, for $M=1$, the gap between the subchannel selection policies $\mathcal{P}_{\nu}$ and $\mathcal{P}_{g_{0}}$ was small in the achieved spectral efficiency, we suggested that the optimal subchannel can be selected based on policy $\mathcal{P}_{g_{0}}$.

The impact of the multiple secondary service users on the total achievable capacity of the secondary network was also studied. It was seen that ignoring the cross interference among the secondary users, the total achievable capacity of the secondary network based on nonuniform subchannel selection with policy $\mathcal{P}_{g_{0}}$ was dramatically increased compared to uniform case. However, considering the cross interference among the secondary users, the decreasing rate of the total achievable capacity of the secondary network in nonuniform subchannel selection is much lower than that of uniform case. This result presented an scaling law of the opportunistic spectrum sharing in DS-CDMA/OFDM systems with multiple users.

\section{ApPEndix A}

\section{Proof of Proposition 1}

Substituting $P_{s j}=\frac{Q_{j}}{g_{0 r_{j}}}$ into (6), and setting $\nu_{r_{j}}=g_{1 r_{j}} / g_{0 r_{j}}$, the maximum achievable capacity $C_{s \mid M}^{g_{0}}$ based on policy $\mathcal{P}_{g_{0}}$ is obtained from $\mathcal{O} 1$ as the following:

Problem $\mathcal{O} 11$.

$$
\begin{array}{r}
C_{s \mid M}^{g_{0}}=\max _{\mathbf{Q}} \sum_{j=1}^{M} B_{c} \int_{\nu_{r_{j}}} \log \left(1+\nu_{r_{j}} \gamma_{Q_{j}}\right) h_{j}\left(\nu_{r_{j}}\right) d \nu_{r_{j}}, \\
\text { s.t. } \quad \sum_{j=1}^{M} \gamma_{Q_{j}}=G N \gamma_{Q}, \quad 0 \leq \gamma_{Q_{j}} \leq G N \gamma_{Q} .
\end{array}
$$

In cases where the subchannel gains $\sqrt{g_{0 r_{j}}}$ and $\sqrt{g_{1 r_{j}}}$ are i.i.d. Rayleigh random variables, $g_{0 r_{j}}$ and $g_{1 r_{j}}$ are exponentially distributed random variables; therefore, using (26) the pdf of $\nu_{r_{j}}, h_{j}\left(\nu_{j}\right)$, is obtained as

$$
\begin{aligned}
h_{j}\left(\nu_{r_{j}}\right)= & \frac{d}{d \nu_{r_{j}}} \int_{0}^{\infty} \int_{0}^{\nu_{r_{j}} g_{0 r_{j}}} N_{j} \sum_{l=0}^{j-1} F_{l}^{j-1} e^{-(N-l) g_{0 r_{j}}} e^{-g_{r_{r_{j}}}} \\
& \times d g_{1 r_{j}} d g_{0 r_{j}}
\end{aligned}
$$

or, equivalently,

$$
\begin{aligned}
h_{j}\left(\nu_{r_{j}}\right)= & \frac{d}{d \nu_{r_{j}}} \sum_{l=0}^{j-1} N_{j} F_{l}^{j-1} \int_{0}^{\infty}\left(1-e^{\left.-g_{0 r_{j} \nu_{r_{j}}}\right)}\right. \\
& \times e^{-(N-l) g_{0_{r_{j}}}} d g_{0 r_{j}}, \\
= & \sum_{l=0}^{j-1} N_{j} F_{l}^{j-1} \int_{0}^{\infty} g_{0 r_{j}} e^{-\left(N-l+\nu_{r_{j}}\right) g_{0 r_{j}}} d g_{0 r_{j}} .
\end{aligned}
$$

Integrating by part yields

$$
h_{j}\left(\nu_{r_{j}}\right)=\sum_{l=0}^{j-1} N_{j} F_{l}^{j-1} \frac{1}{\left(N-l+\nu_{r_{j}}\right)^{2}} .
$$

Substituting (74) into (73) and calculating the integral completes the proof.

\section{APPENDIX B}

\section{Proof of Proposition 2}

Substituting $P_{s j}=\frac{Q_{j}}{g_{0 r_{j}}}$ into (6), and setting $\nu_{r_{j}}=g_{1 r_{j}} / g_{0 r_{j}}$, the maximum achievable capacity $C_{s \mid M}^{g_{1}}$ based on policy $\mathcal{P}_{g_{1}}$ is obtained from $\mathcal{O} 1$ as following:

Problem $\mathcal{O} 12$.

$$
\begin{array}{r}
C_{s \mid M}^{g_{1}}=\max _{\mathbf{Q}} \sum_{j=1}^{M} B_{c} \int_{\nu_{r_{j}}} \log \left(1+\nu_{r_{j}} \gamma_{Q_{j}}\right) h_{j}\left(\nu_{r_{j}}\right) d \nu_{r_{j}}, \\
\text { s.t. } \quad \sum_{j=1}^{M} \gamma_{Q_{j}}=G N \gamma_{Q}, \quad 0 \leq \gamma_{Q_{j}} \leq G N \gamma_{Q} .
\end{array}
$$

In cases where the subchannel gains $\sqrt{g_{0 r_{j}}}$ and $\sqrt{g_{1 r_{j}}}$ are i.i.d. Rayleigh random variables, $g_{0 r_{j}}$ and $g_{1 r_{j}}$ are exponentially distributed random variables; therefore, using (40) the pdf of $\nu_{r_{j}}, h_{j}\left(\nu_{j}\right)$, is obtained as

$$
\begin{aligned}
h_{j}\left(\nu_{r_{j}}\right)= & \frac{d}{d \nu_{r_{j}}} \int_{0}^{\infty} \int_{0}^{\nu_{r_{j}} g_{0 r_{j}}} N_{j} \sum_{l=0}^{N-j} F_{l}^{N-j} e^{-(l+j) g_{r_{j}}} e^{-g_{0 r_{j}}} \\
& \times d g_{1 r_{j}} d g_{0 r_{j}} \\
= & \frac{d}{d \nu_{r_{j}}} \sum_{l=0}^{N-j} \frac{N_{j} F_{l}^{N-j}}{l+j} \int_{0}^{\infty}\left(1-e^{-g_{0 r_{j}} \nu_{r_{j}}(l+j)}\right) \\
& \times e^{-g_{0 r_{j}}} d g_{0 r_{j}} \\
= & \sum_{l=0}^{N-j} N_{j} F_{l}^{N-j} \int_{0}^{\infty} g_{0 r_{j}} e^{-\left(1+\nu_{r_{j}}(l+j)\right) g_{0 r_{j}}} d g_{0 r_{j}} .
\end{aligned}
$$

Integrating by part yields

$$
h_{j}\left(\nu_{r_{j}}\right)=\sum_{l=0}^{N-j} N_{j} F_{l}^{N-j} \frac{1}{\left((l+j) \nu_{r_{j}}+1\right)^{2}} .
$$

Substituting (76) into (75) and calculating the integral proves the proposition. 


\section{REFERENCES}

[1] J.M. Peha, "Approaches to Spectrum Sharing," IEEE Comm. Magazine, vol. 43, no. 2, pp. 10-12, Feb. 2005.

[2] S. Haykin, "Cognitive Radio: Brain-Empowered Wireless Comm.," IEEE J. Selected Areas in Comm., vol. 23, no. 2, pp. 201220, Feb. 2005

[3] Q. Zhao and B. Sadler, "A Survey of Dynamic Spectrum Access: Signal Processing, Networking, and Regulatory Policy," IEEE Signal Processing Magazine, vol. 24, no. 3, pp. 79-89, May 2007.

[4] M.G. Khoshkholgh, K. Navaie, and H. Yanikomeroglu, "Impact of the Secondary Service Transmit Power Constraint on the Achievable Capacity of Spectrum Sharing in Rayleigh Fading Environment," IEEE Comm. Letters, vol. 12, no. 12, pp. 865-867, Dec. 2008.

[5] M.G. Khoshkholgh, K. Navaie, and H. Yanikomeroglu, "On the Impact of the Primary Network Activity on the Achievable Capacity of Spectrum Sharing over Fading Channels," IEEE Trans. Wireless Comm., vol. 8, no. 4, pp. 2100-2111, Apr. 2009.

[6] M.G. Khoshkholgh, K. Navaie, and H. Yanikomeroglu, "Adaptive Multiple Time-Scale Power Allocation for Spectrum Sharing DSCDMA Networks," Proc. IEEE Int'l Conf. Comm. (ICC CogNet '08), pp. 466-470, May 2008.

[7] A.J. Viterbi, CDMA: Principles of Spread Spectrum Communication. Addison-Wesley, 1995.

[8] M. Gastpar, "On Capacity Under Receive and Spatial SpectrumSharing Constraints," IEEE Trans. Information Theory, vol. 53, no. 2, pp. 471-487, Feb. 2007.

[9] S.A. Jafar and S. Srinivasa, "Capacity Limits of Cognitive Radio with Distributed and Dynamic Spectral Activity," IEEE J. Selected Areas in Comm., vol. 25, no. 3, pp. 529-537, Apr. 2007.

[10] A. Ghasemi and E.S. Sousa, "Fundamental Limits of SpectrumSharing in Fading Environments," IEEE Trans. Wireless Comm., vol. 6, no. 2, pp. 649-658, Feb. 2007.

[11] L. Zhang, Y.C. Liang, and Y. Xin, "Joint Beamforming and Power Allocation for Multiple Access Channels in Cognitive Radio Networks," IEEE J. Selected Areas in Comm., vol. 26, no. 1, pp. 38-51, Jan. 2008.

[12] H. Su and X. Zhang, "Cross-Layer Based Opportunistic MAC Protocols for QoS Provisionings over Cognitive Radio Wireless Networks," IEEE J. Selected Areas in Comm., vol. 26, no. 1, pp. 118129, Jan. 2008

[13] S. Geirhofer, L. Tong, and B.M. Sadler, "Cognitive Medium Access: Constraining Interference Based on Experimental Models," IEEE J. Selected Areas in Comm., vol. 26, no. 1, pp. 95-105, Jan. 2008.

[14] M. Sharma, A. Sahoo, and K.D. Nayak, "Channel Selection under Interference Temperature Model in Multi-Hop Cognitive Mesh Networks," Proc. IEEE Int'l Symp. New Frontiers in Dynamic Spectrum Access Network (DySPAN '07), pp. 133-136, Apr. 2007.

[15] N. Nie and C. Comaniciu, "Adaptive Channel Allocation Spectrum Etiquette for Cognitive Radio Networks," Proc. IEEE Int'l Symp. New Frontiers in Dynamic Spectrum Access Network (DySPAN '05), pp. 331-335, Nov. 2005.

[16] D. Tse and P. Viswanath, Fundamentals of Wireless Communication. Cambridge Univ. Press, Sept. 2004.

[17] A. Papoulis and S.U. Pillai, Probability, Random Variables, and Stochastic Processes, fourth ed. McGraw-Hill, 2002.

[18] P. Gupta and P.R. Kumar, "The Capacity of Wireless Networks," IEEE Trans. Information Theory, vol. 46, no. 2, pp. 388-404, Mar. 2000.

[19] M. Grossglauser and D.N.C. Tse, "Mobility Increases the Capacity of Ad Hoc Wireless Networks," IEEE/ACM Trans. Networking, vol. 10, no. 4, pp. 477-486, Aug. 2002.

[20] R. Knopp and P.A. Humblet, "Information Capacity and Power Control in Single-Cell Multiuser Comm.," Proc. IEEE Int'l Conf. Comm. (ICC'95), pp. 331-335, June 1995.

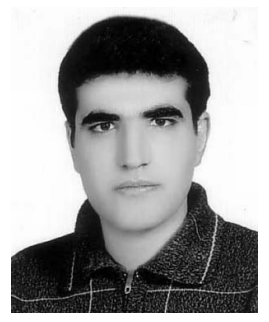

Mohammad G. Khoshkholgh received the BSc degree in electrical engineering from Isfahan University, Isfahan, Iran, in 2006 and the MSc degree in electrical engineering from the Tarbiat Modares University, Tehran, Iran, in 2008. He is currently working toward the $\mathrm{PhD}$ degree at Tarbiat Modares University, Tehran, Iran. His research interests include wireless communications, radio resource allocations, and spectrum sharing. He is a student member of the IEEE.

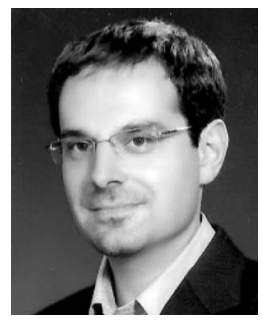

Keivan Navaie received the BSc degree from Sharif University of Technology, Tehran, Iran, the MSc degree from the University of Tehran, Tehran, Iran, and the PhD degree from Tarbiat Modares University, Tehran, Iran, all in electrical engineering in 1995, 1997, and 2004, respectively. From March to November 2004, he was with the School of Mathematics and Statistics, Carleton University, Ottawa, Canada, as a postdoctoral research fellow. From December 2004 to September 2006, he was with the Broadband Communication and Wireless System Centre, Carleton University, Ottawa, Canada. Since September 2006, he has been an assistant professor in the School of Electrical and Computer Engineering, Tarbiat Modares University, Tehran, Iran. $\mathrm{He}$ is also the founder and director of the Wireless Innovation Laboratory (WIL), Tarbiat Modares University, Tehran, Iran. He has been a member of technical committee of WASA 2006, IEEE WCNC 2008, the 16th Iranian Conference on Electrical Engineering (ICEE 2007), IEEE GlobeCom 2008, IEEE WiMob 2008, IEEE WCNC 2009, CNSR 2009, IEEE WCNC 2010, and VTC-Fall 2010. His research interests are mainly in the resource management issues of cellular and multihop wireless networks, spectrum sharing, and performance evaluation. $\mathrm{He}$ is a member of the IEEE.

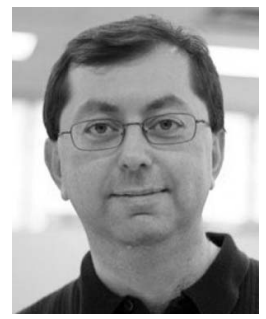

Halim Yanikomeroglu received the BSc degree in electrical and electronics engineering from the Middle East Technical University, Ankara, Turkey, in 1990, the MASc degree in electrical engineering (now electrical and computer engineering) and the $\mathrm{PhD}$ degree in electrical and computer engineering from the University of Toronto, Canada, in 1992 and 1998, respectively. He was with the R\&D Group of Marconi Kominikasyon A.S., Ankara, Turkey, from 1993 to 1994. Since 1998, he has been with the Department of Systems and Computer Engineering at Carleton University, Ottawa, where he is now an associate professor. His research interests include many aspects of the physical, medium access, and networking layers of wireless communications. His research is currently funded by Samsung (SAIT, Korea), Huawei (China), Communications Research Centre Canada (CRC), and NSERC. He was a recipient of the Carleton University Research Achievement Award in 2009. He has been involved in the steering committees and technical program committees of numerous international conferences and has given 17 tutorials in such conferences. He is a member of the steering committee of the IEEE Wireless Communications and Networking Conference (WCNC) and has been involved in the organization of this conference over the years, including serving as the technical program cochair of WCNC 2004 and as the technical program chair of WCNC 2008. He is the general cochair of the IEEE Vehicular Technology Conference to be held in Ottawa in September 2010 (VTC2010-Fall). He was an editor for the IEEE Transactions on Wireless Communications (2002-2005) and IEEE Communications Surveys \& Tutorials (2002-2003), and a guest editor for the Wiley Journal on Wireless Communications \& Mobile Computing. He was an officer of IEEE's Technical Committee on Personal Communications (chair: 2005-2006, vice-chair: 2003-2004, secretary: 2001-02) and was also a member of the IEEE Communications Society's Technical Activities Council (2005-2006). He is also an adjunct professor at King Saud University, Riyadh, Saudi Arabia. He is a member of the Carleton University Senate and a registered professional engineer in the province of Ontario, Canada. He is a member of the IEEE. 